\title{
Two-hop Relay LTE System Using Basic Sequential Algorithmic Scheme and Interference Control
}

\author{
Maryam Hajjar, GhadahAldabbagh \\ Computer Science Department, Faculty of Computing and Information Technology, King Abdulaziz University \\ Jeddah, Kingdom of Saudi Arabia
}

\begin{abstract}
In very crowded areas, a large number of LTE users contained in a single cell will try to access services at the same time causing high load on the Base Station (BS). Some users may be blocked from getting their requested services due to this high load. Using a two-hop relay architecture will help reduce the load from the BS and enhance the capacity of the system. A clustering approach must be used to configure the nodes in such two layer topology. In this paper, clustering of nodes is done based on the Basic Sequential Algorithmic Scheme (BSAS) along with proper frequency allocation and power control in order to avoid excessive interference. The implemented scheme is compared with the case of no relay architecture in terms of capacity enhancement.
\end{abstract}

Key words: LTE, clustering, BSAS, frequency reuse

\section{INTRODUCTION}

LTE has been increasingly deployed worldwide to keep pace with the increasing demand of high data rates from a large number of users. As LTE is being more and more convenient, crowded events such as Hajj, conferences, and matches could get congested with massive LTE users. This congestion in a limited area will cause the Base Station (BS) to be overloaded and thus it will not be able to serve all users successfully. Femtocells and Micro cells have been introduced to reduce the burden of the Macro cell. However, such solutions require high cost due to the additional infrastructure [1].

This paper aims at providing a way to create small cells called clusters inside an LTE macro cell without the need of additional infrastructure. These clusters will be created based on promoting a specific user as a Cluster-Head $(\mathrm{CH})$ to act as a temporal relay station. This $\mathrm{CH}$ will relay all other users' data that are in the same cluster to the BS. These cluster members are called slaves.

In this manner, decisions about the specific allocation of users into different clusters is required, and therefore clustering techniques should be adopted .The allocation of users must also consider appropriate power control and resource allocation in order to mitigate interference and achieve better performance through efficient use of the available spectrum[3].Thus, clustering techniques should be adjusted to meet these requirements.

Clustering is a broad methodology known as the "division of data into groups of similar objects. Each group, called a cluster, consists of objects that are similar between themselves and dissimilar to objects of other groups [4]."
BSAS (Basic Sequential Algorithmic Scheme) is a sequential clustering approach that is simple, yet efficient. It performs a single pass or very few passes on the data set which make it computationally inexpensive for real time applications.

Following the BSAS clustering approach, nodes are clustered into small groups of slaves and a single $\mathrm{CH}$. Only $\mathrm{CHs}$ are directly connected to the base station and act as access points. Each slave node is connected to a $\mathrm{CH}$ node and can use it as a relay to send and receive information to the BS. The group of a $\mathrm{CH}$ node and its associated slaves is called a cluster. Proper allocation of $\mathrm{CH}$ and slave nodes must be determined to meet the constraint of low power clusters. Transmitting and receiving in such low power clusters will enable reusing the frequency in other clusters without causing significant interference and hence capacity is increased.

\section{RELATED WORK}

Clustering Techniques have been used mainly in the fields of data mining and image processing. They have also been widely applied in wireless communications. Two of the most known clustering approaches are the K-means and the Hierarchical Agglomerative Clustering (HAC).Implementations of these two approaches can be found in Wireless Sensor Networks (WSN), Ad-hoc networks and LTE networks.

An example of clustering in LTE is the study in[5]. This study uses two-hop relay system and forms low power usage clusters. Communications inside the small cells (clusters) is done through another frequency band (white space). The study uses K-means clustering algorithm to configure the nodes into clusters. The algorithm assumes a threshold for the maximum number of nodes inside each cluster. Clustering is iterated with the objective of minimizing total power. Using the additional white-space band has the advantage of increasing resources and eliminating the need to partition the LTE band between two layers. Studies $[6,7,8,9,10,11]$ all addressed the use of white-space in LTE. However, the use of such low frequency band (white-space) will cause the signal to propagate for long distances and may result in unexpected interference. Some other studies in LTE and cellular networks $[2,12,13,14]$ use selection strategies to enable the relaying system rather than clustering. In this manner each node chooses its best relay (i.e. $\mathrm{CH}$ ) from a predefined pool of candidate relays. The selection can be based on different criteria. Some are based on choosing the relay with least distance while others are based on the minimum 
path-loss. Using path-loss is better than distance because it gives better estimation of the link quality. These techniques are less complex and more applicable to distributed systems.

As for WSNs, clustering techniques have been widely implemented to solve problems of lifetime and scalability of the network by preserving energy of the sensor nodes. $\mathrm{K}$-means is one of the most used clustering algorithms in WSN $[15,16,17,18,19,20]$. In [15] both a centralized and a distributed approaches of K-means are given. K-means run first by defining a specific number of clusters. Then after all clusters are formed, $\mathrm{CHs}$ are chosen from each cluster based on two factors; distance from cluster centroid and residual energy. In [16]the BPK-means protocol is presented where a specific number of clusters $\mathrm{K}$ is defined based on certain criteria, and an average Ave is defined to be the number of nodes in each cluster as the total number of nodes $\mathrm{N}$ divided by $\mathrm{K}$. Then $\mathrm{K}$-means will run normally with the alteration of the chosen centroids. In each iteration the centroid will be chosen as the actual node that is nearest to the location of the cluster's center. After the completion of K-means, load balancing will start where all clusters will be modified in order to have a total number of nodes equal to Ave. At the last step, $\mathrm{CHs}$ are chosen from each cluster at different rounds based on the same two factors as [15]. The study in [17] also uses K-means to cluster a WSN. It focuses on the $\mathrm{CH}$ selection strategy with the objective of minimizing the consumed energy to increase network life time. After forming the clusters, the node that is nearest to the centroid is chosen as a $\mathrm{CH}$. In each round the energy of the $\mathrm{CH}$ is checked. If it was found to be less than a specific threshold, then the next node in terms of distance to centroid is elected as the new $\mathrm{CH}$ and so on.

Hierarchical Agglomerative Clustering has also been used in WSNs[20, 21, 22, 23]. When using this scheme, there should be no predefined number of clusters. It starts by assuming each node as a single cluster, then work in a bottom-up approach to connect nodes together based on proximity, putting them in a new larger cluster. The algorithm continues merging clusters until all nodes are in a single cluster with a dendrogram tree topology. The algorithm can be applied to disjoint clustering problems by assuming either a specific distance or a number of clusters threshold to stop the process of further merging. This bottom up approach helps to implement a self-organization WSNs. [21] compares between two different methods of calculating the distance between two different clusters in HAC, namely the single link and the complete link methods. In the single link, distance will be measured based on the minimum distance between a node in one cluster and a node in another while in complete link distance will be based on the maximum distance. Results show that the single link is less efficient compared to the complete link. In [22], a distributed HAC is presented. In this study, the received signal strength of nodes is used when location data is not available. In a similar study[23], a predefined threshold is used to cut the hierarchical cluster tree based on transmission radius. Another threshold is defined for the minimum cluster size, clusters that are smaller than this threshold will merge with their closest cluster. $\mathrm{CHs}$ are chosen based on two parameters. Lower level in the hierarchy tree and energy level.
A Hybrid clustering algorithm that is based on both Kmeans and HAC is proposed in[20]. In this approach HAC is used first to define the total number of clusters. This number is then used as $\mathrm{k}$ to run the K-means algorithm. Finally, CHs are defined as the nodes closest to the centroids in all clusters.

In this paper, the BSAS clustering approach will be implemented for LTE. The Algorithm will be modified to accommodate resource allocation and power control specifications.

The rest of the paper is organized as follows. Section 3 explains system model. The proposed algorithm is defined in section 4. Simulation setup and results are given in section 5. Finally, the paper is concluded in section 6 .

\section{SySTEM MODEL}

The system is composed of an LTE BS and a number of users that try to access the BS. Users are classified into two layers. First layer users are directly connected to the BS. Second layer users use one of the first layer users as a relay to access the BS. This kind of association forms a cluster. Each cluster has only one cluster-head $(\mathrm{CH})$ which is a first layer user and a number of slaves that connect to this cluster-head. Each user has a different SINR requirement (a) depending on the type of service needed and the number of associated slaves. CHs will have an aggregated SINR requirement of all its slaves 'SINR values. For simplicity, a uniform SINR threshold is assumed to be $10 \mathrm{~dB}$ for all users own data. A total of 100 Resource Blocks (RBs) are used throughout the cell. Each connected user will be allocated a single RB in a single sub-frame. First, the BS will use all the available Resource Blocks. Then it will start reusing under three conditions:

- A single RB cannot be reused inside one cluster.

- $\mathrm{A} \mathrm{RB}$ used by a $\mathrm{CH}$ cannot be reused in any of its slaves.

- Choose least used RB each time to ensure load balancing.

Path-loss value between a user and its $\mathrm{CH}$ or between a user and the BS will be calculated according to the simple path-loss model:

$$
\mathrm{L}_{\mathrm{ji}}=\mathrm{r}_{\mathrm{ji}}^{\mathrm{e}} 10^{\frac{\xi_{\mathrm{ji}}}{10}}
$$

$r$ is the distance between two nodes $i$ and $j$. e is the path-loss exponent, and $\xi$ is the shadowing value between $i$ and $j$. Least Path-loss value is chosen when trying to connect a user to a $\mathrm{CH}$ or to the $\mathrm{BS}$.

\section{Power Control}

Interference in this work is assumed to be the summation of powers of all other signals that use the same RB as the target signal.

$$
I_{i}=\sum_{\substack{k=1 \\ k \neq i}}^{n} \frac{P_{k}}{L_{i k}}
$$


This equation is used to compute interference for user $\mathrm{i}$, where $\mathrm{P}$ and $\mathrm{L}$ are the power and path-loss of a user $\mathrm{k}$ using the same RB as user $\mathrm{i}$.

In order to reuse $\mathrm{RBs}$ from the first layer, it is important minimize interference so that an acceptable SINR could be achieved. Thus, power control must be applied on all users that access the same RB, i.e. minimizing their power so that they cause less interference to each other while achieving their target SINR. When trying to connect a user with a specific RB that has been reused a number of times, power must be adjusted for all nodes that use the same RB. If, however, this affects the SINR threshold for any of them, then a different association must be selected for this user. In order to evaluate this we must solve a system of linear equations as follow:

From the SINR threshold equation

$$
\frac{\frac{P_{t x, i}}{L_{j i}}}{\sum_{\substack{k=1 \\ k \neq 1}}^{n} \frac{P_{t x, k}}{L_{j k}}+N}=a_{i}, i=1,2, \ldots, n \text { and } j=1,2, \ldots, m
$$

In order to achieve the value of the SINR threshold for all users accessing the same RB, a number of equations must be solved:

$$
\begin{aligned}
& \frac{P_{t_{x}, 1}}{a_{1} L_{11}}-\frac{P_{t_{x}, 2}}{L_{21}}-\frac{P_{t_{x}, 3}}{L_{31}}-\cdots-\frac{P_{t_{x, n}}}{L_{1 n}}=N \\
& -\frac{P_{t x, 1}}{L_{12}}+\frac{P_{t x, 2}}{a_{2} L_{22}}-\frac{P_{t x, 3}}{L_{32}}-\cdots-\frac{P_{t x, n}}{L_{2 n}}=N \\
& -\frac{P_{t x, 1}}{L_{13}}-\frac{P_{t x, 2}}{L_{23}}+\frac{P_{t x, 3}}{a_{3} L_{33}}-\cdots-\frac{P_{t x, n}}{L_{3 n}}=N \\
& -\frac{P_{t x, 1}}{L_{m 1}}-\frac{P_{t x, 2}}{L_{m 2}}-\frac{P_{t x, 3}}{L_{m 3}}-\cdots+\frac{P_{t x, n}}{a_{n} L_{m n}}=N
\end{aligned}
$$

This is a linear equation system and can be written as:

$$
\left[\begin{array}{cccc}
\frac{1}{\mathrm{a}_{1} \mathrm{~L}_{11}} & -\frac{1}{\mathrm{~L}_{21}} & \ldots & -\frac{1}{\mathrm{~L}_{1 \mathrm{n}}} \\
-\frac{1}{\mathrm{~L}_{12}} & +\frac{1}{\mathrm{a}_{2} \mathrm{~L}_{22}} & \ldots & -\frac{1}{\mathrm{~L}_{2 \mathrm{n}}} \\
\vdots & \vdots & \vdots & \vdots \\
-\frac{1}{\mathrm{~L}_{\mathrm{m} 1}} & -\frac{1}{\mathrm{~L}_{\mathrm{m} 2}} & \ldots & +\frac{1}{\mathrm{a}_{\mathrm{n}} \mathrm{L}_{\mathrm{mn}}}
\end{array}\right]\left[\begin{array}{c}
\mathrm{P}_{\mathrm{tx}, 1} \\
\mathrm{P}_{\mathrm{tx}, 2} \\
\vdots \\
\mathrm{P}_{\mathrm{tx}, \mathrm{n}}
\end{array}\right]=\mathrm{N}\left[\begin{array}{c}
1 \\
1 \\
\vdots \\
1
\end{array}\right]
$$

Assuming that the matrices are A,P, and $\mathrm{B}$ respectively, this can be solved using:

$$
\text { A. } P=B \Leftrightarrow P=A^{-1} B
$$

If a solution is not found, then we know that the system with the current number of users accessing the same RB is not achievable and a reduction of this number is needed.

\section{Proposed Algorithm}

Associations is based on reducing power whilst having a fair channel condition. Fair channel condition is achieved when the target SINR of the user's requested service is met. If the target SINR is not met, user will blocked from getting the service. In order to meet these constrains, slaves will connect to $\mathrm{CHs}$ based on finding the minimum path-loss. This will help reducing the power and increasing the gain to the whole system. Moreover, the maximum number of $\mathrm{CHs} /$ candidate $\mathrm{CHs}$ is chosen to be 100 as this is the total number of RBs. The term $\mathrm{CH}$ will be used to indicate a first layer user in general as it is a candidate for this rule. The BSAS clustering schemes is detailed as follows:

1. Let:

$\mathrm{C}$ be the set of clusters/cluster-heads

$\mathrm{N}=\{\mathrm{n} 1, \mathrm{n} 2, \mathrm{n} 3, \ldots .$.$\} set of nodes, all current active$ users.

$\mathrm{K}=100$ maximum no. of clusters (the case of 100 $\mathrm{RBs}$ )

$\Theta$ (dissimilarity threshold) as the maximum accepted path-loss between a slave and a ClusterHead.

2. Sort $\mathrm{N}$ based on path-loss to the BS

3. assign first node $\mathrm{n} 1$ as a Cluster-Head,

4. for every node $\mathrm{n}$ from $\mathrm{i}=2$ to $|\mathrm{N}|$,

find cluster ch from $\mathrm{C}$ such that has min path-loss to $\mathrm{n}$.

if Pathloss(n, ch) $>\Theta$ AND $(|C|<K)$

- Create a new cluster

- Update Clusters

else

Add $\mathrm{n}$ to the nearest cluster ch that satisfy conditions of reuse.

\section{Simulation Setup and Results}

In this section, an evaluation of the performance of the algorithm is done. Also, the simulation model and results are being described and compared to an LTE cell with no relays. The simulation environment is designed using MATLAB. The assumptions for the simulation study are as follows.

Table 1: Parameters used in simulation

\begin{tabular}{|l|l|}
\hline Parameter & Value \\
\hline Cell radius & $100 \mathrm{~m}$ \\
\hline Cell area & $31,400 \mathrm{~m}^{2}$ \\
\hline Base Frequency & $2 \mathrm{GHz}$ \\
\hline Effective Bandwidth (B) & $18 \mathrm{MHz}$ \\
\hline Required Throughput (R) & $64 \mathrm{Kbps}$ (VoIP service) \\
\hline No. of Resource Blocks & 100 \\
\hline $\begin{array}{l}\text { Number of nodes (n) /sub- } \\
\text { frame }\end{array}$ & $150,200,250,300$ \\
\hline Number of nodes (N) /frame & $1500,2000,2500,3000$ \\
\hline Number of iterations & 100 \\
\hline Path-loss exponent & 4 (shadowed urban) \\
\hline Shadowing & $6 \mathrm{~dB}$ \\
\hline Position of BS (x,y) & $(0,0)$ \\
\hline Noise & $\sim-200 \mathrm{dBm}$ \\
\hline SINR th & $10 \mathrm{~dB}$ \\
\hline Max Tx Power UE & 0.796 Watts, 29 dBm \\
\hline Max no. of slaves & 5 \\
\hline Duplexing & FDD \\
\hline Modulation scheme & 64 QAM \\
\hline Scheduling & Round Robin \\
\hline
\end{tabular}

- $1 \mathrm{RB}$ is allocated for each user's data.

- Each user will have a single sub-frame in each frame in a round robin fashion. 
- VoIP service is assumed only with the requirement of $64 \mathrm{Kbps}$, thus SINR threshold is computed as follows:

$$
\begin{aligned}
& \text { SINR }=2^{\frac{N R}{B}}-1 \\
& \text { SINR }=10 \mathrm{~dB}
\end{aligned}
$$

- Required SINR for each user is $10 \mathrm{~dB}$.

- The simulation environment is composed of a single BS and $n$ user nodes.

- The BS is fixed and located at the center of the cell.

- The locations of user nodes are randomly distributed in the cell area.

- The BS can gather information about users.

- All user nodes are mobile and have limited movement due to density.

- A maximum density of 1 person/m2 is assumed. Thus, the maximum total population is approximately 30,000.

- Each user occupies 1 resource block (RB) for a fraction of time: 6 mins / hour (activity 10\%)

- Maximum active users: $10 \%$ of $30,000=3000$.

- Simulation is done to check the resource allocation during a single sub-frame (of duration $1 \mathrm{~ms}$ )that is supposed to be accessed by $10 \%$ of all users (due to the abovementioned considered user activity pattern).

A total of $100 \mathrm{RBs}$ are used at a single sub-frame, and the scheduling requirement will be fixed to $1 \mathrm{RB}$ per user. This will give a total of 100 direct connections to the BS. Each of the n-100 remaining users could be either a second layer users or blocked users depending on the availability of resources and system interference condition.

\section{Acknowledgement}

This paper has been funded by the National Plan for Science, Technology and Innovation (MAARIFAH) King Abdulaziz City for Science and Technology - the Kingdom of Saudi Arabia - award number

(12-INF 2743-03). The authors also, acknowledge with gratitude the Science and Technology Unit in King Abdulaziz University for technical support.

\section{REFERENCES}

[1] A. Khalifah, N. Akkari, and G. Aldabbagh, "Dense Areas Femtocell Deployment: Access Types and Challenges," presented at the 2014 Third International Conference on eTechnologies and Networks for Development (ICeND), Beirut, Lebanon, 2014.

[2] H. Nourizadeh, S. Nourizadeh, R. Tafazolli,"Performance Evaluation of Cellular Networks with Mobile and Fixed Relay Station," Vehicular Technology Conference, 2006. VTC2006 Fall. 2006 IEEE 64th, pp.1,5, 25-28 Sept. 2006.

[3] I. Ahmed, A. Mohamed, "On the joint scheduling and intracell interference coordination in multi-relay LTE uplink," Globecom Workshops (GC Wkshps), 2012 IEEE, vol., no., pp.111,115, 3-7.

[4] O. Abu Abbas, "Comparison Between Data Clustering Algorithms," the International Arab Journal of Information Technology, Vol.5, No. 3, July 2008
[5] H. Tabrizi, H. G. Farhadi, J.M. Cioffi, "CaSRA: An algorithm for cognitive tethering in dense wireless areas," Global Communications Conference (GLOBECOM), 2013 IEEE, vol., no., pp.3855,3860, 9-13 Dec. 2013 doi: 10.1109/GLOCOM.2013.6831674

[6] N. Akkari, G. Aldabbagh, M. Nahas, B. Bawazeer, J. Cioffi, and H. Tabrizi, "Coordinated Tethering Over Cellular Networks: Handover Scenarios and Signaling," presented at the 2013 IEEE 24th International Symposium on Personal Indoor and Mobile Radio Communications (PIMRC), London, United Kingdom, 2013.

[7] N. Akkari, G. Aldabbagh, M. Nahas, and J. Cioffi, "Dynamic Clustering Protocol for Coordinated Tethering over Cellular Networks," Journal of Network and Computer Applications, vol. 42, pp. 92-101, 06/2014.

[8] H. Tabrizi, G. Farhadi, J. Cioffi, and G. Aldabbagh, "Coordinated Tethering over White-Spaces," IEEE Transactions on Vehicular Technology, vol. PP, p. 1, 03/10/2014 2014.

[9] G. Aldabbagh, S. T. Bakhsh, N. Akkari, S. Tahir, H. Tabrizi, and J. Cioffi, "QoS-Aware Tethering in a Heterogeneous Wireless Network using LTE and TV White Spaces," Computer Networks, 13th of February 2015.

[10] G. Aldabbagh, T. Bakhsh, N. Akkari, S. Tahir, S. Khan, and J. Cioffi, "Distributed Dynamic Load Balancing in a Heterogeneous Network using LTE and TV White Spaces," Wireless Networks, 12th of February 2015.

[11] U. Al-Haddad, G. Aldabbagh, and N. Dimitriou, "Clustering over TV White Space in Dense Wireless Areas: Dynamic Hotspot Selection and Resource Allocation" MitteilungenKlosterneuburg, vol. 65, 2015.

[12] H. Nourizadeh, R. Tafazolli“'Capacity improvement of WCDMA cellular systems through different relaying strategies", MWCN 2005, Marrakech, Morocco, 19 September.

[13] V. Sreng, H. Yanikomeroglu, D.DFalconer, "Relayer selection strategies in cellular networks with peer-to-peer relaying," Vehicular Technology Conference, 2003. VTC 2003-Fall. 2003 IEEE 58th, vol.3, no., pp.1949,1953 Vol.3, 6-9 Oct. 2003

[14] H. Wei, H. Jing, W. Haiming, "UL Performance Evaluation of Relay Enhanced FDD LTE-AdvancedNetworks," Wireless Communications, Networking and Mobile Computing (WiCOM), 2011 7th International Conference on , vol., no., pp.1,5, 23-25 Sept. 2011

[15] P. Sasikumar,S. Khara, "K-Means Clustering in Wireless Sensor Networks," Computational Intelligence and Communication Networks (CICN), 2012 Fourth International Conference on, vol., no., pp.140,144, 3-5 Nov. 2012

[16] T. Liansheng,G. Yanlin,C. Gong, "A Balanced Parallel Clustering Protocol for Wireless Sensor Networks Using KMeans Techniques," Sensor Technologies and Applications, 2008. SENSORCOMM '08. Second International Conference on , vol., no., pp.300,305, 25-31 Aug. 2008

[17] Y. P. Geon, et al, "A Novel Cluster Head Selection Method based on K-Means Algorithm for Energy Efficient Wireless Sensor Network," Advanced Information Networking and Applications Workshops (WAINA), 2013 27th International Conference on , vol., no., pp.910,915, 25-28 March 2013

[18] A. Talgini, V. Shakarami,F. Sheikholeslam, A. Chatraei, "Aerial node placement in wireless sensor networks using 
Maryam Hajjar et al., International Journal of Emerging Trends in Engineering Research, 8(9), September 2020,5764 - 5768

Fuzzy K-means clustering," e-Commerce in Developing Countries: With Focus on e-Trust (ECDC), 2014 8th International Conference on, vol., no., pp.1,7, 24-25 April 2014

[19] H. Harb, A. Makhoul,D. Laiymani, A. Jaber, R. Tawil, "Kmeans based clustering approach for data aggregation in periodic sensor networks," Wireless and Mobile Computing, Networking and Communications (WiMob), 2014 IEEE 10th

International Conference on, vol., no., pp.434,441, 8-10 Oct. 2014

[20] G. Kumar, H. Mehra, A.R. Seth, P. Radhakrishnan, N. Hemavathi, S. Sudha, "An hybrid clustering algorithm for optimal clusters in Wireless sensor networks," Electrical, Electronics and Computer Science (SCEECS), 2014 IEEE Students' Conference on , vol., no., pp.1,6, 1-2 March 2014
[21] L. Chung-Horng, Z. Chenjuan, "Using Hierarchical Agglomerative Clustering in Wireless Sensor Networks: An Energy-Efficient and Flexible Approach," Global Telecommunications Conference, 2008. IEEE GLOBECOM 2008. IEEE, vol., no., pp.1,5, Nov. 30 2008-Dec. 42008

[22] T.K.Jain, D.S. Saini, S.V. Bhooshan, "Performance analysis of hierarchical agglomerative clustering in a wireless sensor network using quantitative data," Information Systems and Computer Networks (ISCON), 2014 International Conference on, vol., no., pp.99,104, 1-2 March 2014

[23] L. Chung-Horng,Z. Chenjuan, "Using Hierarchical Agglomerative Clustering in Wireless Sensor Networks: An Energy-Efficient and Flexible Approach," Global Telecommunications Conference, 2008. IEEE GLOBECOM 2008. IEEE, vol., no., pp.1,5, Nov. 30 2008-Dec. 42008 